\title{
Kubas Gesetzesdekret Nr. 50/1982 - Castros Kniefall vor dem Kapitalismus?
}

\author{
von Ludwig Gramlich
}

\section{Einleitung}

1. Von der größten Insel in der Karibik kam unlängst wieder einmal überraschende Kunde: Ein Gemeinwesen, das durch verfassungskräftige, solenne Proklamation den "Imperialismus als schlimmsten Feind der Völker" verdammt ${ }^{1}$ und dessen direkte oder auch mittelbare "Interventionen" in die inneren wie die äußeren Angelegenheiten eines jeden Staates, zugleich alle Formen wirtschaftlichen Zwangs und der Einmischung - ja schon der Drohung mit solchem Vorgehen - in die "staatliche Integrität und die politischen, ökonomischen und kulturellen Wesensbestandteile der Nationen" entschieden ablehnt, ${ }^{2}$ sollte der Inlandstätigkeit auch ausländischer Privat-Unternehmen fürs Erste eher mißtrauisch gegenüberstehen, von ihr die Unterminierung der eigenen Staats, Gesellschafts-, Wirtschaftsordnung befürchten. Kubas Leitungsorgane halten es nichtsdestotrotz nunmehr nicht allein im Interesse der wirtschaftlichen Entwicklung des eigenen Landes für geboten, fremde Gesellschaften auf kubanischem Gebiet wirken zu lassen (so die dritte Erwägung im Vorspruch des legislativen Dekrets Nr. 50). Sie bezeichnen derartige Aktivitäten gar als eine Hilfe zur Stärkung des sozioökonomischen Systems, vorausgesetzt nur, die (institutionalisierte) Zusammenarbeit werde vom sozialistischen Staat frei vorangetrieben oder doch immerhin akzeptiert (vgl. die vierte Präambel-Erwägung).

Der solchermaßen im Vorspruch des legislativen Dekrets Nr. 50 "über wirtschaftliche Vereinigungen zwischen kubanischen und ausländischen Einrichtungen" vom 15. Februar $1982^{3}$ dokumentierte Anschauungswandel rührt freilich kaum von ungefähr. Zum einen bürdeten der Verfall des Zuckerpreises sowie der ähnlich rapide Rückgang der Erlöse für Nickel, das zweitwichtigste Rohstoffexportprodukt der Insel, nicht zuletzt jedoch auch die erst allmählich wieder nachgebenden Zinssätze auf den Finanzmärkten der westlichen Industriestaaten und im Bereich der Xenodevisen der mittelamerikanischen Republik binnen weniger Jahre eine beträchtliche Schuldenlast in konvertiblen Währungen auf, deren akuter Druck zu einem förmlichen Gesuch des Banco Nacional

1 Constitution de la Republica de Cuba (17./24. Febr. 1976), Art. 12 (a); Text und engl. Úbersetzung in Bd. IV der "Constitutions of the World", hrsg. von A. P. Blaustein/G. H. Flanz ("Cuba" bearb. von P. S. Falk).

2 Ebd., Art. 12 (b). S. dazu etwa Comment (L. B. Klein), The Socialist Constitution of Cuba (1976), Colum. J. Transnat. L. 17 (1978) 451, 475.

3 Vom 15. Febr. 1982; engl. Ubersetzung (durch die kubanische Camara de Comercio) in deren Broschüre "Possibility of Joint Ventures in Cuba" vom Febr. 1982, 9-13 (= I.L.M. 21 (1982) 1107-1111). 
um Zahlungsaufschub und Umstrukturierung, verbunden mit einer vorläufigen Einstellung von Tilgungsleistungen führte. ${ }^{4}$ Andererseits erlangte Kuba ungeachtet seiner Bereitschaft zum Abschluß von Globalentschädigungsabkommen mit mehreren Staaten, deren Angehörige während der castristischen Revolution Expropriationsmaßnahmen unterworfen worden waren, ${ }^{5}$ kaum offizielle Entwicklungshilfe aus dem westlichen Ausland, ${ }^{6}$ insonderheit aber keine von multilateralen Finanzierungsinstitutionen. ${ }^{7}$ Auch im internationalen Rahmen wußten nämlich die USA ihr beträchtliches politisches Gewicht geltend zu machen, so daß im Einklang mit dem stets noch andauernden umfassenden Wirtschaftsembargo der westlichen Supermacht ${ }^{8}$ die Zuckerinsel von jeglicher Unterstützung bei ihrer wirtschaftlichen Entwicklung abgeschnitten geblieben wäre, hätten nicht die Mitgliedsstaaten des RGW, ${ }^{9}$ an ihrer Spitze die UdSSR, massiven Beistand geleistet, über dessen uneigennützigen Charakter allerdings Zweifel angebracht erscheinen. ${ }^{10}$

2. Trifft es demnach zu, daß Kuba, "um in der kapitalistischen Welt wettbewerbsfähig zu sein, ein bißchen mehr kapitalistisch (habe) werden müssen "? ${ }^{11}$ Eine Analyse der neuen Vorschriften für ausländische Investitionen vermag diese Aussage eines leitenden

4 Näheres hierzu bsp. in Financial Times vom 2.6. 1982 ("A further tightening of the screw ") und vom 3. 3. 1983 ("Cuba secures agreement on rescheduling of $\$ 413 \mathrm{~m}$ debt “); Frankfurter Rundschau vom 1. 9.1982 ("Fidel Castro bereitet Kubaner auf längere Durststrecke vor"); Neue Zürcher Zeitung vom 11. 9.1982 ("Chronisch passiver Außenhandel Havannas"), vom 5. 3. ("Kubas Gläubigerländer zur Umschuldung bereit") und vom 20. 4. 1983 ("Kubas Umschuldung noch nicht unter Dach"); vgl. aber auch den "Economic Report" des Banco Nacional de Cuba vom August 1982.

5 S. die Übereinkunft mit der Schweiz vom 2. März 1967 (BBI. I, 936) und dazu L. Caflisch. Schw. Jb. Int. R. 25 (1968) 235, 259-260; sowie die Konvention mit Frankreich vom 16. März 1967 (s. Anwendungsdekret Nr. 67-853 vom 20. Sept. d. J., J. O. 4. 10., 9761).

6 So gilt Kuba etwa nicht als Entwicklungsland im Sinne des deutschen Gesetzes über steuerliche Maßnahmen zur Förderung von privaten Kapitalanlagen in Entwicklungsländern (i. d. F. vom 21. Mai 1979, BGBI. I, 564) - fehlt sein Name doch in der Ausschließlichkeit beanspruchenden Auflistung des $\S 6$ - und kommt daher auch nicht in den Genuß von Mitteln via Förderung von Niederlassungen deutscher Unternehmen in Entwicklungsländern (vgl. die Richtlinien des Bundesministers für wirtschaftliche Zusammenarbeit vom 27. Juni 1979, BAnz. Nr. 133 vom 20. Juli 1979, 3, unter I.1.b)).

7 Sowohl die Bretton Woods-Institutionnen wie auch die Interamerikanische und die Karibische Entwicklungsbank fördern zufolge ihrer Satzung allein die (weniger entwickelten) Mitgliedsstaaten (vgl. nur Art. 1 (i), 3 (1)(a) der I.B.R.D.-Statuten, U.N.T.S. 2 (1947) 134, BGBI. 1952 II, 664; Art. 1 (1), 3 (1) des I.A.D.B.-Gründungsübereinkommens, U.N.T.S. 389 (1961) 72, BGBI. 1976 II, 38).

8 S. die Darstellung bei $B$. Lindemeyer, Schiffsembargo und Handelsembargo (1975); zu den "Cuban Assets Control Regulations" (31 C.F.R. Part 515) vgl. etwa Nielsen v. Secretary of the Treasury, 424 F. 2d 833 (D. C. Cir. 1970), und Real v. Simon, 510 F. 2 d 557 (5th Cir. 1975); s. a. A.J.I.L. 64 (1970) 951-953, 70 (1976) 357-358.

9 Kuba gehört dem COMECON seit 1972 an (s. R. Bystricky, Le droit de l'intégration économique socialiste (1979) 47-48).

10 Vgl. nur NZZ vom 10. 3. 1983 ("Kubas Wirtschaft im 25. Revolutionsjahr") und die Kritik von B.-O. Bryde, Der Kodex über Auslandsinvestitionen der Sozialistischen Republik Vietnam, VRU 11 (1978) 103, 105 mit Nr. 16, an D. Senghaas, Weltwirtschaftsordnung und Entwicklungspolitik (1977) 21. Ähnliches mag gelten in bezug auf die Darstellungen von T. Meier, Ist Kubas Wirtschaft bankrott?, Antiimperialistisches Informationsbulletin 1/1983, 12, 13, und von H. März, Zum II. Parteitag der KP Kubas, Deutsche Außenpolitik 26 (1981) H. 3, 25, 27-29.

11 Zitat laut Financial Times vom 2. 6. 1982 (oben, Nr. 4). 
Offiziellen kaum zur Gänze zu bestätigen, wenngleich die kubanische Regelung im Vergleich zu denjenigen anderer sozialistischer Staaten durchaus einige bemerkenswerte Eigenheiten aufweist.

\section{Einzelheiten des neuen kubanischen Investitionsregimes}

1. Den inhaltlichen Rahmen des "decreto-ley" habe sich Kuba von Ungarn abgeschaut, urteilte ein hiesiger Wirtschaftsjournalist, nachdem die "vorsichtige Offnung für Auslandskapital" stattgefunden hatte. ${ }^{12}$ Rein äußerlich kann von solcher Ähnlichkeit allerdings kaum die Rede sein, hebt sich doch das Gesetzesdekret Nr. 50 sowohl dem Umfang - zusammen mit Sonder- und Schlußbestimmungen enthält es 50 Artikel - wie auch dem Aufbau und der Systematik nach deutlich vom vorgeblichen Modell ab. ${ }^{13}$ In fünf Kapiteln wird zunächst die Rechtsstellung der wirtschaftlichen Vereinigungen näher bestimmt (insbesondere in Art. 1 (2), 4-12); weiterhin finden sich Vorschriften betr. finanzielle (Art. 14-24), Handels- (Art. 31-35) und Beschäftigungsaspekte (Art. 36-44) in bezug auf die Betätigung aller zulässigen Arten ökonomischer Assoziierung, ${ }^{14}$ und im zentralen Kapitel III sind die Besteuerungsmodalitäten festgelegt (Art. 25-29). Inzwischen sind auch die in verschiedenen Normen des Regelungswerks (z. B. in Art. 10, 26, 44) vorgesehenen Ausführungsbestimmungen ergangen, so daß einem reibungslosen Vollzug eigentlich nichts Entscheidendes mehr im Wege stehen sollte.

2. Bei näherem Zusehen weist das kubanische Regime für ausländische Investitionen freilich noch etliche Unklarheiten und wirft es sohin Zweifelsfragen auf, deren baldige Beantwortung einem verbesserten Investitionsklima ${ }^{15}$ nur dienlich sein könnte.

12 G. Kornat, Handelsblatt vom 26. 7. 1982 ("Kuba: Vorsichtige Offnung für Auslandskapital").

13 Basierend auf einer Ermächtigung in §31 der Verordnung mit Gesetzeskraft Nr. 19/1970 (vom 7. Aug. d. J.) des Präsidialrates der Volksrepublik (Ungarn) über die wirtschaftlichen Assoziationen (deutsche Úbersetzung in: RIW/AWD 1973, 126-129), erging am 3. Okt. 1972 das finanzministerielle Dekret Nr. 28/1972 über wirtschaftliche Vereinigungen mit ausländischer Beteiligung (deutsch ebd., 124-126; engl. in I.L.M. 12 (1973) 989-994; dazu etwa F. Madl, Die Neuregelung wirtschaftlicher Assoziationen mit ausländischer Beteiligung in Ungarn, RIW/AWD 1973, 121-124). In I.L.M. 17 (1978) 1451-1455 findet sich in englischer Úbersetzung eine Neufassung, welche Änderungen insbesondere durch die Verordnung Nr. 7/1977 berücksichtigt.

14 Nach Art. 1 (2) des Gesetzesdekrets sind neben den genannten gemeinsamen Unternehmen mit kubanischer und ausländischer Kapital-Beteiligung, eigener Rechtspersönlichkeit wie sächlicher Ausstattung auch verschiedene andere Formen wirtschaftlichen Zusammenschlusses erlaubt, die nicht die Einrichtung einer körperschaftlichen Organisation bedingen. Ähnlich verhält es sich nach Maßgabe von $\S 4$ sowie den Kap. 2 und 3 des bulgarischen Staatsrats-Ediktes Nr. 535 vom 25. März 1980 betr. wirtschaftliche Zusammenarbeit zwischen inländischen juristischen und fremden juristischen wie natürlichen Personen (engl. in I.L.M. 19 (1980) 992-1002); vgl. dazu W. G. Kuiper, Bulgaria: New Joint Venture Legislation, Eur. Tax. 20(1980) 293, 295; D. Pfaff, Vertragsgestaltung gemischter Gesellschaften in Bulgarien, RIW/AWD 1981, 668, 670. In Ungarn gilt hingegen ein recht strikter Typenzwang (Art. I des zuvor genannten Dekrets 1972/77).

15 Vgl. vorerst L. G. Nehrt, The Political Climate for Private Foreign Investment (1970); jüngst auch J. Voss, The Protection and Promotion of Foreign Direct Investment in Developing Countries: Interests, Interdependencies, Intricacies, I.C.L.Q. 31 (1982) 686-708. 
a) Ein erstes Problem liegt dabei bereits im Bereich der sektoralen Beschränkung (potentieller) ausländischer Investoren. ${ }^{16}$ Das Gesetzesdekret kennt zumindest im operativen Teil keine Vorschrift, die bestimmte Zweige der kubanischen Volkswirtschaft für Auslandskapital generell und absolut zugänglich machte. Hieraus zu schließen, derartige Schranken existierten sonach überhaupt nicht, dürfte jedoch kaum der Teleologie des Regelungsganzen gerecht werden. Schon in der dritten Erwägung des Vorspruchs heißt es nämlich einschränkend, das Land bedürfe ausländischer Assoziationspartner für jene Tätigkeiten, die nach mehr finanziellen Mitteln, Rohstoffen, Technologien und Märkten verlangten, als eigenständig verfügbar seien, um Kubas natürliche und menschliche Ressourcen auszuschöpfen; auch werden in der fünften Präambel-Zeile als wesentliche Ziele gegenwärtiger Außenwirtschaftspolitik Exportsteigerung und ausländischer Tourismus exemplarisch aufgeführt (vgl. auch Art. 2, 18 (1), Sondervorschriften 1 und 2). Durch diese Manifestation der gesetzgeberischen Absichten dürften die Befugnisse der Genehmigungsbehörde (Art. 1 (1), (3) ) bereits ab initio begrenzt und kanalisiert sein, wie der (erneute) Bezug auf eine Eignung des ausländischen Beitrags zur Förderung der Landesentwicklung belegt. Im übrigen erhellt aus einem Vergleich nationaler Gesetzgebungen für ausländische Investitionen, daß ungeachtet der sozioökonomischen und/oder systemaren Unterschieden geschuldeten Besonderheiten im einzelnen selbst die dem privaten Unternehmertum aufs Entschiedenste verpflichteten westlichen Industriestaaten gewisse strategische bzw. sensitive Bereiche ihrer Volkswirtschaft entweder gänzlich hoheitlicher Eigenbetätigung ${ }^{17}$ oder doch zumindest ausschließlich den eigenen Staatsangehörigen vorbehalten. ${ }^{18}$ Wenn daher die kubanische Verfassung in Art. 15 die Eigentumsverhältnisse festschreibt, nicht zuletzt auch näher darlegt, welche Wirtschaftszweige und -einrichtungen unabänderlich sozialistisches Staatseigentum sind - d. h. dem ganzen Volk, aber auch nur diesem, gehören -, weiterhin eine eingehendere Regelung von Struktur, Aufgabe und Befugnissen staatlicher Unternehmungen durch Gesetz anordnet (Art. 17), ${ }^{19}$ stellt sich ausländische (private) Aktivität im Geltungsbereich dieses Grund-Gesetzes noch stets als eng begrenzte, punktuelle Ausnahme dar. Daß etwa auch Investitionen im (Nickel-)Bergbau, in Agroindustrieprojekten oder in (Verkehrs-) Infrastrukturvorhaben möglich wären, wird zwar zuweilen in der westlichen Wirtschaftspresse erwähnt, entbehrt aber einer triftigen Begründung. Deren Genehmigung

16 Dazu allgemein etwa D. Carreau/T. Flory/P. Juillard, Droit international économique $\left({ }^{2} 1980\right)$ 477-479; H.J. Hahn/L. Gramlich, Regelungsprobleme grenzüberschreitender Investitionen, ArchVR 21 (1983) 145-238.

17 Hierzu rechneten etwa im Geltungsbereich des Grundgesetzes insonderheit die Verwaltungs- und Finanzmonopole (dazu BVerfG vom 14. Jan. 1976, E 41, 205, 217-218); vgl. weiterhin W. H. Balekjian, Legal Aspects of Foreign Investment in the European Economic Community (1967) 26-31.

18 Dies geschieht in Gestalt einer Ausnahme von der grundsätzlich festgelegten Inländergleichbehandlung für "Investitionen" etc. von Angehörigen der anderen staatlichen Vertragspartei (Art. II (1), (2)) selbst in dem wahrhaft umfassenden Kapital-Schutz-Vertrag, den die U.S.A. und Ägypten am 29. Sept. 1982 in Washington abschlossen; s. Art. II (3) i.V.m Anhang, wo diverse Bereiche insbesondere des Dienstleistungssektors aufgeführt sind (I.L.M 21 (1982) 927-949).

19 Dazu auch Klein, Colum. J. Transnat. L. 1978, 484-487. 
steht vielmehr wohl nur in seltenen Fällen zu erwarten, ${ }^{20}$ und von einem Rechtsanspruch hierauf ist nirgends in der Regelung die Rede, was in Anbetracht des Fehlens fest umrissener materieller Voraussetzungen für eine positive Entscheidung der Kommission auch schwerlich verwundern kann.

b) Uber Bedingungen und Verfahren in bezug auf die Vornahme einer ausländischen Investition enthält das legislative Dekret auch ansonsten nicht immer hinreichend eindeutige Regeln. Die eigentliche Genehmigungsbehörde, genannt "Kommission", ist dem Exekutivausschuß des Ministerrats unterstellt; ihre Mitglieder werden von diesem Regierungsgremium benannt (Art. 1 (1) der Gesetzesverordnung), in Einklang mit dessen konstitutionellem Kompetenzen-Katalog. ${ }^{21}$ Daneben statuiert Art. 10 des Investitionsregimes als Bedingung für die Verbindlichkeit jeder Assoziierungsabrede deren Eintragung in ein spezielles Register bei der Camara de Comercio; dieser Akt kann sinnvollerweise wohl erst nach der Kommissionserlaubnis erfolgen, was auch die systematische Stellung der Vorschrift - im Anschluß an die Bestimmungen über den organisatorischen Rahmen der internationalen Unternehmenszusammenarbeit (Art. 7-9) - offenbart.

Die Funktion der Kommission nun, insbesondere das ihrer Regelung überantwortete Rechtsverhältnis, wird nun allerdings recht verwirrend gekennzeichnet: Vorab Art. 16 (1), 17 (a) handeln von der Genehmigung der Errichtung eines gemeinsamen Unternehmens oder von anderen Formen ökonomischer Assoziierung. Demgegenüber verlautet jedoch in Art. 1 (1), die Kommission sei befugt, staatlichen Unternehmen und anderen nationalen Organisationen den Zusammenschluß mit ausländischen Investoren auf kubanischem Territorium zu gestatten. Art. 2 ermöglicht dem Ministerrats-Exekutivausschuß schließlich, die Kommission zu ermächtigen, über die Zulässigkeit bestimmter als Kapital anzusehender Leistungen von Staatsunternehmungen an die körperschaftlichen Vereinigungen befinden zu können. Aus den Bestimmungen des legislativen Dekrets geht nicht klar hervor, ob die zuerst genannte Verfahrensmodalität in der Tat zwei aufeinander folgende Stufen der Genehmigungsprozedur aufrichtet, nämlich zunächst lediglich die Aushandlung des Assoziationsvertrages betreffend, nach dessen

20 Ähnlich Financial Times vom 2. 6. 1982 (oben, Nr. 4) und The Economist vom 19./25. 2. 1983 ("Big is not so bad after alla); s. aber auch Handelsblatt vom 26. 7. (oben, Nr. 12) und Frankfurter Rundschau vom 1. 9. 1982 (oben, Nr. 4).

S. im Vergleich etwa die Auflistung der für ausländische Investitionen offenen Wirtschaftszweige in Art. I (1), (2) des Staatsrats-Dekrets Nr. 424 vom 2. Nov. 1972 über die Errichtung, Organisation und Betätigung gemischter Gesellschaften in der Sozialistischen Republik Rumänien (engl. Ubersetzung in I.L.M. 12 (1973) 651-656), in Art. I (1), (2) sowie im Anhang (Art. 1) der polnischen Ministerrats-Entschliel.3ung Nr. 24 vom 7. Febr. 1979 (hierzu J. Scriven, Joint Ventures in Poland, J.W.T.L. 14 (1980) 424, 429; s. jetzt auch $W$. Bergmann, Reform des Wirtschafts- und Außenwirtschaftsrechts in Polen, RIW/A WD 1982, 798, 800, zur Erweiterung des zugänglichen Tätigkeitsspektrums durch Art. 2 des Gesetzes vom 6. Juli 1982), oder in Art. 10 des jugoslawischen Gesetzes über Investitionen von Ressourcen ausländischer Personen in inländische Organisationen vereinigter Arbeit vom 30. März 1978 (engl. Text in I.L.M. 18 (1979) 230-247; vgl. etwa $P$. Le Tourneau. L'autogestion et la Loi du 30 mars 1978 sur les investissements étrangers en Yougoslavie, Dr. prat. comm. int. 6 (1980) 385, 404).

21 Vgl. Art. 96 (d), (j), (o), (p) der Verfassung von 1976, und dazu Klein. Colum. J. Transnat. L. 1978, 507. 
Fixierung seitens der Vereinigungspartner sodann eine Inhaltskontrolle durch staatliche Behörden. Daß wohl eher allein über den Antrag des kubanischen Investitionsbeteiligten zu entscheiden ist - dem bereits die Entwürfe von Assoziationsabrede und -statuten (bei gemeinsamen Unternehmen) zugrundeliegen -, dürfte aus den detaillierten normativen Vorgaben in Art. 8 (2), (3) zum einen, dem Wortlaut des Art. 16 (1) andererseits zu folgern sein, welch letzterer nurmehr eine zusätzliche Möglichkeit bezeichnet. ${ }^{22}$

Ebenso zweifelhaft erscheint die Einordnung der Ausnahmebewilligung nach Art. 15 in den Ablauf des Genehmigungsverfahrens: Bedarf es etwa bei hälftiger oder gar mehrheitlicher ausländischer Kapitalzufuhr ${ }^{23}$ hiernach (auch) eines Gesuchs des fremden Partners an das Ministergremium, prüft dieses allein das Vorliegen eines exzeptionellen Sachverhalts, wie und wann wird hier (daneben) die Kommission tätig? Daß der Exekutivausschuß gegenüber dem Vollzugsorgan generell weisungsbefugt ist, besagt ja nicht ohne weiteres, er dürfe auch unbesehen in dessen Zuständigkeiten nach außen hin eintreten. ${ }^{24}$

c) Was weiterhin die Stellung der Genehmigungsbehörde anbelangt, so weicht die kubanische Regelung für den Normalfall zumindest von den entsprechenden Vorschriften anderer sozialistischer Investitionsregimes $a b,{ }^{25}$ betraut sie doch, hierin einem

22 Ebenso die offiziöse Kommentierung seitens der Camara de Comercio in "Possibility . . ." (a.a.O. (Nr. 3) 4: "Method of Approval“): Hiernach werde die Kommission von Beginn an von einschlägigen Initiativen Kenntnis haben (von wem?), aber erst - binnen eines Zeitraums von zwei Monaten - über den endgültigen Vereinigungs-Vorschlag befinden, dessen Aushandlung auf kubanischer Seite allein Sache der Unternehmensvertreter sei. Während nun die hier genannte Entscheidungsfrist immerhin in Art. 17 (b) des Gesetzesdekrets, wenngleich in anderem Zusammenhang, förmlich niedergelegt ist, dürften die weiteren Darlegungen freilich nicht unbesehen als normative Äul.serung gelten können, sowohl in Anbetracht ihrer Herkunft wie der Art der Kundgabe!

Im übrigen bedarf auch in Ungarn nach Art. 3.(1), (3) i. V. m. Art. 2 des Dekrets von 1972/77 offenbar formell erst der bereits fixierte Gesellschaftsvertrag der Genehmigung. Ebenso verhält es sich wohl in der Volksrepublik China (Art. 3 des Gesetzes über gemeinsame Unternehmen mit chinesischen und ausländischen Investitionen vom 1./8. Juli 1979; engl. Text in I.L.M. 18 (1979) 1163-1165; deutsch auch bei $R$. Heuser, Das Joint Venture-Gesetz der Volksrepublik China vom Juli 1979, RIW/AWD 1979, 797-802; s. aber auch die Analyse von C. Torem/J. Wohl, China's Law on Joint Ventures, Dr. prat. comm. int. 6 (1980) 47, 58-59, betr. vorherige (informelle) Kontakte). Nicht deutlich gefaß3t sind schließlich auch Art. 17, 18 des Regimes für ausländische Investitionen in der Sozialistischen Republik Vietnam vom 18. Apr. 1977 (französische Ubersetzung in VRU 11 (1978) 107-112); in einer Note hierzu (Harv. I.L.J. 19 (1978) 681, 684 mit $\mathrm{Nr}$. 18) unterstreicht $S$. Hickok, wesentliche Entscheidungen im Hinblick auf Organisation und Kontrolle des Investitionsvorhabens oder -Unternehmens blieben individuellen Verhandlungen überlassen, die (auch nach Ansicht dieser Verf.) notwendig bereits vor dem Antrag auf Genehmigung nach Art. 17 zu führen sein werden.

23 Die zunächst ausnahmslos geltende Obergrenze von $49 \%$ fremder Beteiligung an Joint Ventures auf dem Territorium sozialistischer Staaten (s. noch Art. 4 des rumänischen Dekrets 1972) wurde im Laufe der Jahre fast allerorten für besondere Fallgestaltungen relativiert (vgl. dazu etwa J. Zoubek, Joint Ventures in East Europe, J.W.T.L. 9 (1975) 427, 429; G. Florescu, Les sociétés mixtes dans les pays socialistes, nouvelle forme de coopération économique internationale, Dr. prat. comm. int. 4 (1978) 243, 252; sowie bsp. Art. 4 (2) der volkschinesischen, Art. 7 (3) der vietnamesischen, Art. 9 (3) der bulgarischen Regelung).

24 Dies dürfte auch e contrario aus Art. 91 (d) der Verfassung 1976 gefolgert werden können (vgl. Klein. Colum. J. Transnat. L. 1978, 505).

$25 \mathrm{Vgl}$. etwa Art. 2 (1), 3 (1) der ungarischen Verordnung: Zuständigkeit des Finanzministers: Art. 5 des bulgarischen Edikts: Ministerrat; Art. 18 des rumänischen Dekrets: Staatsrat: Art. 19 des vietnamesischen 
spezifisch lateinamerikanischen Brauch folgend, ${ }^{26}$ mit dieser Aufgabe nicht ein oberstes Staatsorgan, sondern ein nachrangiges Gremium (die "Kommission"). Uber deren personelle Zusammensetzung verlautet im Dekret freilich nichts, obgleich mutmaßlich Organwalter bzw. Behörden der Außenwirtschaftsverwaltung und der jeweils betroffenen Fachressorts in ihr vertreten sein dürften. ${ }^{27}$ Auch wenn aber die Kommissionsmitglieder im Ministerrang stehen sollten, änderte sich wohl nichts an dem untergeordneten Status des Gesamtorgans.

Ein zusätzliches Indiz für solche Bewertung stellt wohl der Inhalt der dieser Stelle übertragenen Eingangskontrolle dar (von Kompetenzen in bezug auf die einmal genehmigte Inlandsbetätigung ${ }^{28}$ ist im Gesetzesdekret nichts zu lesen!): Die Prüfung erstreckt sich vor allem auf die Erfüllung der in den Abreden der Kooperationspartner gemäß Art. 8, 9 festzulegenden Regeln organisatorischer und operativer Art, bleibt also weithin formal und korrigiert insonderheit schwerlich den Inhalt der einvernehmlich gefundenen Konditionen des Zusammenwirkens Punkt für Punkt.. ${ }^{29}$ Auch beim Gebrauchmachen

Regimes: Außenhandelsminister. S. aber auch Art. 3 des chinesischen Joint Venture-Gesetzes, welches ebenfalls eine Kommission für ausländische Investitionen und eine gesonderte Eintragung bei der Allgemeinen Industrie- und Handelsverwaltung kennt, sowie die durchaus ähnliche Regelung in Art. 4, 10 (1), $40 \mathrm{ff}$. des jugoslawischen Gesetzes 1978 (dazu näher Z. Pouch, Das neue Investitionsrecht Jugoslawiens, RIW/ AWD 1978, 429, 433).

26 Vgl. z. B. die argentinischen Bestimmungen seit 1976 (Gesetz 21.382 vom 13. Aug. d. J. (engl. in I.L.M. 15 (1976) 1364/1367-1371; s. a. die Neufassung durch Gesetz 22.208 (1980) in: I.C.S.I.D., Investment Laws of the World I, Argentina, 3-8), Art. 4, 6 i. V. m. Art. 33 ff. der Durchführungsverordnung 283/77 (engl. in I.L.M. 16 (1977) 660-676) bzw. Art. 38 ff. des Dekrets 103/81 (I.L.W., a.a.O., 9-23) und dazu G. C. McKinnis, The Argentine Foreign Investment Law of 1976, Colum. J. Transnat. L. 17 (1978) 357, 370-378; ferner Art. 2, 22 ff. des bolivianischen Gesetzesdekrets Nr. 07366 vom 20. Okt. 1965 über Förderung und Anreiz für sowie Zusammenarbeit mit ausländischen Investitionen (engl. in I.L.M. 5 (1966) 208-217) und Art. 42 ff. der Gesetzesverordnung Nr. 10045 vom 10. Dez. 1971 über Investitionen (engl. in I.L.M. 11 (1972) 375-377); Art. 3 ff. des venezolanischen Dekrets Nr. 63 vom 28. Apr. 1974 (engl. in I.L.M. 13 (1974) 1221-1234). S. a. G. Foeth, Investitionen in Lateinamerika (1979) 85-86 (Ekuador), 129-130 (Chile) u. ö.

27 So jedenfalls in vergleichbaren Einrichtungen anderswo; s. etwa Art. 11 der venezolanischen Verordnung 1974; Art. 27 des chilenischen Gesetzesdekrets 600 vom 13. Juli 1974 (engl. in I.L.M. 13 (1974) 1176-1188) und Art. 13 des Statuts für ausländische Investitionen (Gesetzesverordnung 1748) vom 11. März 1977 (engl. in I.L.M. 17 (1978) 134-138); sowie Foeth, a.a.O. (Nr. 26) 167-169 (zu Art. 11 des mexikanischen Investitionsgesetzes vom 7. Febr. 1973, engl. in I.L.M. 12 (1973) 643-649).

28 Was zunächst als Regelungslücke - im Verhältnis zu Vorschriften wie denjenigen Ägyptens (vgl. Art. 26 des Gesetzes Nr. 43 betr. arabische und ausländische Kapitalinvestitionen und Freizonen vom 19. Juni 1974, engl. in I.L.M. 13 (1974) 1500-1512) oder der Philippinen (Art. 16 des Republic Act No. 5186 vom 16. Sept. 1967, engl. in I.L.M. 6 (1967) 1174-1193) - erscheint, hat offensichtlich System, findet sich doch eine besondere Kompetenz in bezug auf ausländisches Kapital überall in sozialistischen Investitionsregimes nur für das Eingangs-/Zulassungsstadium begründet. Da dies auch und gerade in zentralverwaltungswirtschaftlich organisierten Gemeinwesen jedoch kaum einen gänzlichen Verzicht auf Beaufsichtigung der Vereinigungstätigkeiten bedeuten kann, liegt wohl die Möglichkeit hoheitlicher Eingriffe aufgrund gemeinen Rechts nicht fern, insbesondere wenn und weil dafür wiederum durchgängig keine spezifischen Voraussetzungen normiert sind (s. jedoch Art. 30, 31 des rumänischen Dekrets 1972). Vgl. auch unten, (bei) Nr. 66, 92.

29 Im Verlauf der Vertragsverhandlungen mag der Gaststaat aber schon durch Direktiven an die Leitung des beteiligten nationalen Unternehmens oder auch durch Einschaltung kompetenter Amtspersonen in die Gespräche o. ä. seine Auffassung von deren wünschenswertem Ausgang in der gebotenen Deutlichkeit dargelegt haben, wodurch auch der ausländsiche Investor mehr Gewilheit über die Realisierbarkeit seiner mittel- und langfristigen Vorstellungen erlangen kann (vgl. Pfaff. RIW /AWD 1981, 617-672, zur vergleichbaren Situation in Bulgarien, sowie oben, $\mathrm{Nr}$. 22). 
von ihren Befugnissen nach Art. 2, 3 und 16, 17 wird die Kommission wohl kaum mehr als bloß administrativ tätig, und ähnlich mag dies schließlich für die Wertung der Genehmigungsfähigkeit einer Vereinigung gelten, die ja nur beim Erschließen gewinnbringender, der Landesentwicklung dienlicher Betätigungsfelder bejaht werden kann (Art. 1 (1)). Haben sich nämlich der kubanische und der ausländische Partner - im Bereich der offenen Sektoren der Volkswirtschaft - auf eine künftige (institutionalisierte) Zusammenarbeit geeinigt, so erscheint es kaum denkbar, daß bei diesem Stand der Dinge die Genehmigung für das inländische Unternehmen (vgl. Art. 6) versagt werden sollte. ${ }^{30}$

3. Wie in allen sozialistisch ausgerichteten Staaten, die sich ausländischem Kapital nicht (mehr) völlig verschließen, ${ }^{31}$ wie aber auch in vielen durchaus marktwirtschaftlich orientierten Entwicklungsländern ${ }^{32}$ ist zur Realisierung ausländischer (Direkt-)Investitionen auf kubanischem Staatsgebiet die vertragliche Abmachung mit inländischen Organismen - nach Art. 5 der Gesetzesverordnung kommen als Partei Staatsunternehmen, Konsortien und andere nationale Organisationen (s. a. Art. 1 (2), 2) in Betracht; ${ }^{33}$ sonach jedenfalls nicht private Individuen per se - eine Grundvoraussetzung. Sie ergibt sich unmittelbar aus dem Verbot totaler ausländischer Kontrolle bzw. Verfügungsmacht über inländische Wirtschaftseinheiten ${ }^{34}$ und ist auch direkt zur Durchsetzung des staatlichen Außenwirtschaftsmonopols vonnöten..$^{35}$ Die Kooperation verläuft auf Unterneh-

30 Immerhin sieht das jugoslawische Gesetz 1978 einen Rechtsbehelf gegen ablehnende Entscheide des Investitionsgremiums vor (Art. 45; s. dazu J. G. Scriven, Yugoslavia's New Foreign Investment Law, J.W.T.L. 13 (1979) 95, 101-102).

31 Obwohl in der DDR, der Tschechoslowakei und der UdSSR bislang keine besonderen Rechtsvorschriften allein über die Zulassung fremder Privat-Investoren bestehen, dürfte dieser Zustand immerhin nicht einem absoluten Verbot gleichzusetzen sein (s. J. Rajski, Les principes du droit commercial international de certains pays socialistes européens, Rec. des Cours 174 (1982-I) 9, 39-58). Anders verhält es sich jedoch de constitutione lata vornehmlich mit Albanien (Art. 28 der Verfassung vom 28. Dez. 1976 (Blaustein/Flanz, a.a.O. (Nr. 1) Bd. I, bearb. von G. H. Flanz) verbietet sowohl eine Kreditaufnahme im Ausland als auch die Zusammenarbeit mit fremden "kapitalistischen" Unternehmen) und mit Nord-Korea (vgl. D. A. Loeber, Foreign Participation in Soviet Enterprises?, Dr. prat. comm. int. 6 (1980) 215, 237).

32 Hierzu zählen etwa die Mitgliedstaaten des Anden-Pakts (nach Maßgabe insbesondere der Art. 38-44 des "Lima-Statuts" - Gemeinsames Regime für die Behandlung ausländischen Kapitals und über Warenzeichen, Patente, Lizenzen und Gebühren -, welches als Entscheidung Nr. 24 der Kommission auf Grund der Art. 26 und 27 des Ubereinkommens von Cartagena vom 26. Mai 1969 (engl. in I.L.M. 8 (1969) 910-939) erging und seither mehrfach geändert wurde; die Fassung zum 30. Nov. 1976 findet sich in engl. Úbersetzung in I.L.M. 16 (1977) 138-158). Vgl. näher etwa Foeth, a.a.O. (Nr. 32) 61-64; C. T. Oliver, The Andean Foreign Investment Code: A New Phase in the Quest for Normative Order as to Direct Foreign Investment, A.J.I.L. 66 (1972) 763, 772-777; P. Schliesser/S. Volnay, Régime des investissements étrangers à l'intérieur du Marché commun andin, J.D.I. (Clunet) 99 (1972) 558, 564-566; P. Schulenburg, Die Behandlung ausländischer Direkt-Investitionen im Andenpakt, RIW/AWD 1975, 395, 397-398.

33 Ähnlich etwa Art. 5 des rumänischen Dekrets 1972; Art. 9 des jugoslawischen Gesetzes 1978.

34 S. im einzelnen etwa W. G. Friedmann/J.-P. Béguin, Joint International Business Ventures in Developing Countries (1971) 10-29.

35 S. dazu Art. 18 der kubanischen Verfassung 1976. Deutlich wird dies z. B. in Art. 3 des bulgarischen Außenhandelsgesetzes vom 20. Nov. 1969 (engl. in I.L.M. 14 (1975) 397-399) und in der entsprechenden Bestimmung des ungarischen Außenwirtschaftsregimes (ebd., 405-415: Art. 3, 4). S. a. M. Kemper/D. Maskow, Außenwirtschaftsrecht der DDR (1975) 147-149. 
mensebene; die an ihr beteiligten (inländischen) staatseigenen Einrichtungen verfügen bereits nach innerstaatlichem Recht regelmäßig nicht über spezifisch hoheitliche Befugnisse, machen hiervon jedenfalls in kommerziellen Beziehungen keinen Gebrauch, so daß sie im Verhältnis zueinander ebenso wie die ausländischen Gesellschaften o. ä. vorab den gründungsvertraglichen Bindungen unterliegen und sich hiervon, wie Art. 11, 12 (1) exemplarisch verdeutlichen mögen, nicht einseitig lossagen können. ${ }^{36}$ Jegliche Streitigkeiten im Innenverhältnis der Partner einer wirtschaftlichen Vereinigung müssen dementsprechend im allseits vereinbarten Verfahren beigelegt werden (Art. 13), eine Formulierung, die Schlichtungs- (vgl. Abs. 2) und Schiedsprozeduren nahelegen dürfte, sogar einen Rekurs zu internationaler Arbitration offenlassen mag, wie ein Vergleich mit Art. 35 (2) zeigen sollte. ${ }^{37}$ Auch wenn die kubanische Verfassung eine funktionale richterliche Unabhängigkeit verbürgt (Art. 125, 123 (c)), wird ein ausländischer Investor wohl schwerlich Abhilfe von nationalen Gerichten erwarten und so kaum je deren Entscheidungszuständigkeit wählen. ${ }^{38}$

4. Allgemeine Grundsätze zur Behandlung einmal ins Werk gesetzter wirtschaftlicher Vereinigungen ${ }^{39}$ enthält das neue kubanische Investitionsregime nicht, abgesehen davon, daß nach Vorschrift des Art. 7 gemeinsame Unternehmen die kubanische Staatsangehörigkeit besitzen ${ }^{40}$ und sie weithin (Art. 6, 12 (1) 2) den Regelungen des Handelsgesetzbuchs unterliegen. ${ }^{41}$ Der ausländische Gesellschafter in seiner vermögenswerten Beteiligung ist so von Rechts wegen wie aus Praktikabilitätsgründen darauf verwiesen, eine kontraktuelle Absicherung seiner Bestandserhaltungs- und Gewinninteressen zu betreiben; offenbar bietet sich ein recht weiter materieller Spielraum, für sachnotwendig erachtete Garantien festschreiben zu können. Allerdings wird davon auszugehen sein,

36 So auch - allerdings bezogen auf Außenwirtschaftsverträge generell - M. Kemper/W. Panzer u. a., Wirtschafts- und Außenwirtschaftsrecht für Okonomen (1977) 499-502; s. ferner Florescu. Dr. prat. comm. int. 1978, 252-253. Die offiziöse Kommentierung bezeichnet sohin wohl mit Recht das einmal errichtete gemeinsame Unternehmen als "gänzlich vom State unabhängig" (a.a.O. (Nr. 3) 4).

37 Durchweg bleiben die Schiedsk lauseln in den "Joint Venture"-Gesetzgebungen recht vage, zumindest was die mögliche Befassung externer Instanzen anbelangt (vgl. Art. 25 der vietnamesischen Regelung 1977; Art. 14 des volkschinesischen Gesetzes 1979; Art. 38 des rumänischen Dekrets von 1972; klarer hingegen Art. 52 des jugoslawischen Gesetzes 1978).

$38 \mathrm{Zu}$ den Gründen s. jetzt H. P. de Vries, International Commercial Arbitration: A Contractual Substitute for National Courts, Tulane L. Rev. 57 (1982/83) 42, 64; vgl. auch R. Patzina, Rechtlicher Schutz ausländischer Privatinvestoren gegen Enteignungsrisiken in Entwicklungsländern (1981) 132.

39 Eindringlich hierzu Carreau/Flory/Juillard, a.a.O. (Nr. 16) 512-521; Hahn/Gramlich. ArchVR 1983, 204-230.

40 Ebenso etwa Art. 3 des rumänischen Dekrets 1972; Art. 7 (2) der vietnamesischen Regelung; in anderen Staaten gilt dies auch ohne ausdrücklichen normativen Ausspruch (vgl. J. Rajski. Basic Principles of International Trade Law of Certain European Socialist States and of East-West Trade Relations, Dr. prat. comm. int., 4 (1978) 9, 26). Damit scheidet aber fürs Erste ein Zugang zu Streitbeilegungsfazilitäten des International Centre for Settlement of Investment Disputes aus, die auch in ihrer Erweiterung durch die Verwaltungsrats-Entscheidung vom Sept. 1978 (s. I.L.M. 21 (1982) 1443-1472) nicht diejenigen Sachverhalte einbeziehen, in denen der eigene Staat als andere Partei belangt werden müßte (vgl. zur Novelle P. Toriello, The Additional Facility of the International Centre for Settlement of Investment Disputes, Ital.YB.Int.L. 4 (1978/79) 59-83).

41 Vergleichbar wohl dem Art. 14 der ungarischen Verordnung von 1972/77. 
daß die Obergrenze des Kapitaleinschusses auch Auswirkungen auf die Besetzung der Vorstandsämter, die Stimmenverteilung in der Gesellschafterversammlung etc. zeitigt, auch hier der ausländische Investor nicht mehr als seinem Beitrag entsprechende Minderheitsrechte innehat. ${ }^{42}$ Inhalte und Schranken organisationsbezogener Bestimmungen finden sich jedoch bindend vorgegeben (Art. 6, 8 (3) a. E.). ${ }^{43}$ So gelten Vorschriften finanziellen Inhalts wie das Gebot der Eröffnung und des Haltens von Konten in konvertiblen Währungen bei inländischen staatlichen Banken (Art. 19) oder in bezug auf eine Kreditaufnahme in Devisen. Eine solche kann grundsätzlich auch bei ausländischen privaten Banken bewerkstelligt werden; darüber hat freilich der Banco Nacional zu wachen, vornehmlich um Erfordernissen währungspolitischer Art genügen zu können (Art. 21) ${ }^{44}$ Recht ungewöhnlich mutet die Bestimmung an, der (potentielle) ausländische Investor könne zu einer auch geldwerten Garantieleistung bereits vor dem rechtsgültigen Zustandekommen der wirtschaftlichen Assoziierung angehalten werden (Art. 16 (1)); nachdem hierfür jedoch einmal Zinsen zu zahlen sind (Abs. 2), zum andern solcher Beitrag entweder mit dem nachmaligen Gesellschaftsanteil verrechnet oder aber alsbald in voller Höhe zurückerstattet werden muß (s. Art. 17), mag diese Regelung durchaus als sachgerechte Vorbeugemaßnahme eines Gaststaats im Hinblick auf ggf. erforderliche, ebenfalls kostenträchtige Vorleistungen der eigenen Unternehmen gewertet werden können. ${ }^{45}$ Dies gilt wohl umsomehr, als das Verlangen, Sicherheit beizubringen, nicht obligatorisch, sondern ins Ermessen der Kommission gestellt ist. Von einigem Gewicht für fremde Investoren dürfte auch die weitere Bestimmung sein, wonach der Bewertungsmaßstab für die jeweiligen (Kapital-)Beteiligungen einer Absprache zwischen den Partnern überlassen ist (Art. 14 (2) a. E.); dabei sind konvertible Währungen - also nicht der kubanische Peso $^{46}$ - zugrundezulegen, wie übrigens bei allen geschäftlichen Operationen mit Auslandsbezügen (Art. 19, 20, 22, 27). ${ }^{47}$

42 Zum Gleichlauf von Kapitalbeteiligung und Einfluß auf die Unternehmenspolitik s. vornehmlich die Bestimmungen im "Lima-Statut" (Art. 1, 31 (c)) sowie Art. 5 (4) des mexikanischen Gesetzes 1973; zu letzterem etwa $M$. W. Gordon, The Contemporary Mexican Approach to Growth with Foreign Investment: Controlled but Participatory Independence (1973), in: K. R. Simmonds/M. W. Gordon, Multinational Corporations Law - Mexico and Central America, Vol. I (1982) B.1., 1, 35-36; W. Frisch/Philipp, Ausländische Investitionen und Grunderwerb durch Ausländer in Mexiko, RIW/AWD 1982, 167, 170-171. Andernorts (s. nur Art. 10 der bulgarischen, Art. 6 der chinesischen Regelung) finden sich hierfür eingehendere Vorschriften, vorab zur Nationalität der Mitglieder der Unternehmensleitungs-Organe.

43 Mithin dürfte es zutreffen, wenn die Kommentierung der Handelskammer (a.a.O. (Nr. 3) 4) den gemeinsamen Unternehmen "Autonomie" zuschreibt und sogar von "absoluter Freiheit" des unternehmerischen Gebarens nach innen wie nach außen spricht (ebd.).

44 Gerade in Lateinamerika sind Restriktionen finanzieller Art nicht eben selten (vgl. bsp. Art. 14, 15, 17 des Lima-Statuts; Art. 40-46 der venezolanischen Verordnung 1974; Art. 17 des argentinischen Gesetzes über ausländische Investitionen 20.557 vom 7. Nov. 1973 (engl. in I.L.M. 12 (1973) 1489-1505).

Dagegen findet Art. 21 Entsprechungen in Bulgarien (Art. 14 des Edikts 1980), der Volksrepublik China (Art. 8 (3) des "Joint Venture"-Gesetzes 1979) und auch in Ungarn (vgl. Art. 10, 11 der Verordnung 1972/77).

45 So die Begründung für die ähnliche Regelung in Polen (s. Bergmann, RIW/AWD 1982, 801).

46 Kuba ist im Jahre 1964 aus dem Internationalen Währungsfonds ausgetreten (s. J. Gold, Membership and Nonmembership in the International Monetary Fund (1974) 342-344).

47 Dies wird noch einmal in der offiziösen Kommentierung unterstrichen (a.a.O. (Nr. 3) 5). Das Gesetzesdekret enthält allerdings keine Verpflichtung für die nationalen Banken, den wirtschaftlichen Vereinigungen stets die 
5. a) Die im engeren Sinne abgabenrechtlichen Regelungen weiterhin - in Kap. III des legislativen Dekrets - zeichnen sich durch ein beachtliches Maß an Flexibilität und Ubersichtlichkeit aus. ${ }^{48}$ Zum einen ist klargestellt, welche fiskalischen Belastungen auf die Wirtschaftsvereinigungen zukommen (Art. 26), aber auch, von welchen Steuern kraft Gesetzes(verordnung) Befreiung eingeräumt wird, nämlich vorab von der allgemeinen Einkommen- und Körperschaftsteuer (Art. 25; Gesetz 998 vom 1. 1. 1967). Auch im Vergleich zu Normen sozioökonomisch verwandter Staaten nimmt sich Kubas Regime recht vorteilhaft aus: Die Besteuerung des Nettojahresgewinns mit einem Satz von 30 \%, wobei insbesondere reinvestierte Beträge, ${ }^{49}$ aber zudem auch alle einvernehmlich getätigten Rücklagen ${ }^{50}$ vom Reingewinn abgezogen werden dürfen, bewegt sich im unteren Bereich der üblichen Festlegungen; ${ }^{11}$ die Vereinigung braucht die Steuer erst in den beiden ersten Monaten des je folgenden Jahres zu entrichten, ohne zuvor Abschlagszahlungen leisten zu müssen; schließlich ist keine zusätzliche Abgabe in bezug auf die Gewinnverteilung an die Unternehmenspartner vorgesehen. ${ }^{52}$ Diesem recht ungeschmälerten Ertragsanspruch bei ausländischen Investoren entspricht es, daß Lohnsummensteuern nur für die kubanischen Beschäftigten anfallen,,$^{53}$ ausländische Fachkräfte, wie sie bei Bedarf verpflichtet und eingesetzt werden dürfen, hingegen keiner inländischen (Einkommen-)Steuerpflicht unterliegen, sondern lediglich der nationalen Einwan-

benötigten Devisen verfügbar zu machen; sohin bleiben letztere vorab auf Eigenmittel oder Auslandskredite angewiesen (so ausdrücklich Art. 24 des rumänischen Dekrets 1972).

48 S. demgegenüber die weitaus umfangreicheren Vorschriften der Volksrepublik China (Gesetz vom 26. Aug. 1980, engl. in I.L.M. 19 (1980) 1452-1453; Durchführungsbestimmungen vom 10. Dez. d. J., I.L.M. 20 (1981) 384-389; vgl. auch die Darstellung von F. Münzel, Das chinesische Steuerrecht für Joint Ventures, RIW/AWD 1981, 24-28).

49 Deren Vorzugsbehandlung ist häufig normiert; s. nur Art. 36 der bulgarischen Regelung 1980; Art. 6 des chinesischen Steuergesetzes für Joint Ventures und Art. 6 der Durchführungsbestimmungen; Art. 4 des rumänischen Dekrets Nr. 425 vom 2. Nov. 1972 (engl. in I.L.M. 12 (1973) 656-657); Art. 7 der ungarischen Verordnung von 1972/77; Art. 10 (5) des vietnamesischen Regimes 1977.

50 Solches Vorgehen ist regelmäßig eher in Industriestaaten anzutreffen (vgl. $R$. Anthoine, Tax Incentives for Private Investment for Developing Countries (1979) 3-5); s. freilich schon Art. 11 bzw. 12 des südvietnamesischen Investitionsrechts vom 14. Febr. 1963 (Gesetzesdekret Nr. 2-63, engl. in I.L.M. 2 (1963) 494-504) resp. vom 2. Juni 1972 (Gesetz Nr. 4/72, engl. in I.L.M. 11 (1972) 882-891).

51 S. etwa für Polen $A$. Burzinski/J. Rajski, Le régime juridique des investissements étrangers en Pologne, Dr. prat. comm. int. 6 (1980) 171, 180-181; Begmann, RIW/AWD 1982, 800; für die Volksrepuboik China Art. 3 (4) des Steuergesetzes 1980, sowie Financial Times vom 13.4. 1983 ("China woos foreign investors"); für Bulgarien Art. 36, 37 des Edikts vom selben Jahr; für Ungarn Art. 4 der Verordnung von 1972/77; sowie in bezug auf nichtsozialistische Entwicklungsländer etwá $H$. K. Singhal, Taxing for Development: Incentives Affecting Foreign Investment in India, Harv.I.L.J. 14 (1973) 50, 56-82; J.-C. Bancal, L'imposition des bénéfices des sociétés étrangères en Arabe Saoudite, Dr. prat. comm. int. 3 (1977) 603-614.

52 So ausdrücklich der Handelskammer-Kommentar (a.a.O. (Nr. 3) 5). Insofern unterscheidet sich das kubanische Regime deutlich von dem anderer sozialistischer Staaten (vgl. nur Art. 37 der bulgarischen Regelung 1980; Art. 4 des chinesischen Steuergesetzes 1980; aber auch Art. 15 (2) des vietnamesischen Regimes von 1977 und zu letzterem Hickok, Harv. I.L.J. 1978, 693).

53 Art. 26 (b) 1 der Gesetzesverordnung. In dieser Abgabe (25\%) - die nach S. 3 offenbar neben dem Lohn abzuführen ist - sind die Sozialversicherungsbeiträge des Unternehmens enthalten (Kommentierung, a.a.O. (Nr. 3) 5), wie es bsp. auch in Ungarn (Art. 4 A: 35 \%) und Rumänien (Art. 35 des Dekrets Nr. 424/1972) der Fall ist, während etwa Bulgarien im Edikt von 1980 ausdrücklich auch ausländische Beschäftigte zu (gegenüber Inländern allerdings niedrigeren : $20 \%$ des Lohns) Leistungen zur Sozialversicherung heranzieht. 
derungs- und Fremden-Gesetzgebung (Art. 43 S. 1). ${ }^{54}$ Ein- und Ausfuhrabgaben, insonderheit also Zölle, werden regelmäßig auch von Wirtschaftsvereinigungen mit ausländischer Beteiligung erhoben; im Bereich des Tourismus sieht das Gesetzesdekret jedoch allgemeine oder doch partielle Ausnahmemöglichkeiten vor (1. Sondervorschrift, (a)), wie im übrigen auch für die Besteuerung generell und ebenso für die im Normalfall geschuldete Kfz-Steuer (vgl. Art. 26 (d)). Wohl mehr der Vollständigkeit halber weist Art. 26 (e) auf die Gebührenpflicht für die Beantragung, Erlangung und/ oder Erneuerung von "Dokumenten" hin; gerade über dieses Instrument scheint freilich mancherorts der gaststaatliche Fiskus erhebliche Beträge einzufordern, ${ }^{55}$ sei es wegen der Entgeltlichkeit einer Vielzahl für die Tätigkeitsaufnahme erforderlicher behördlicher Erlaubnisse oder aber wegen der Höhe der Kostenforderung im Einzelfall. ${ }^{56}$ Immerhin gilt auch in Kuba diese Verpflichtung durchgängig; weder die Sonderregelung im Hinblick auf "Gebiete von großer Bedeutung für den internationalen Tourismus" noch die für jede Art der Assoziierung anwendbare Befreiungsvorschrift des Art. 28 lassen auch insoweit Abweichungen zugunsten von Wirtschaftsvereinigungen mit ausländischer Beteiligung zu. Gerade die zuletzt angeführte Norm ermöglicht jedoch weitreichende zeitliche und sachliche Steuerbefreiungen bzw. -vergünstigungen, bei deren Festlegung sich das zuständige staatliche Komitee für Finanzen an dem Nutzen auszurichten hat, den ein Unternehmen für die Volkswirtschaft erbringen dürfte; ${ }^{57}$ zulässig sind allerdings nur ihrer Dauer nach begrenzte, temporäre Exemtionen, ${ }^{58}$ dies jedoch erforderlichenfalls in Höhe der gesamten sonst geschuldeten Gewinnsteuern oder Grenzabgaben. ${ }^{59}$

b) Ungeklärt ist derzeit noch, ob und wie die kubanischen fiskalischen Anreize dem ausländischen Investor eine endgültige Steuerersparnis bescheren (können). In Erman-

54 Dies dürfte nicht zuletzt der offiziöse Kommentar hinreichend klarstellen (a.a.O. (Nr. 3) 5, 7). Demgegenüber hat die Volksrepublik China im Jahre 1980 (26. Aug.) gebietsansässige Ausländer vornehmlich hinsichtlich ihrer Beschäftigung in einem "Joint Venture" der Einkommensteuerpflicht unterworfen (engl. Ubersetzung des Gesetzes in I.L.M. 19 (1980) 1451-1452, der Ausführungsvorschriften vom 10. Dez. d. J. in I.L.M. 20 (1981) 389-392), und auch die UdSSR hat vor nicht allzu langer Zeit ähnliche Normen aufgerichtet (s. das Dekret des Präsidiums des Obersten Sowjet vom 12. Mai 1978, engl. in I.L.M. 17 [1978] 1108-1114, und dazu J. E. Martinez, Soviet Personal Taxation of Foreigners, Colum.J.Transnat.L. 19 [1981] 455-492; $W$. Bergmann. Neuregelung der Besteuerung von Ausländern in der UdSSR, RIW/AWD 1978, 520-522).

$55 \mathrm{Vgl}$. z. B. M. A. G. Caballero, Taxation of Foreign Investments in Spain - Direct Investment, Eur. Tax. 23 (1983) 1, 7-8; mexikanisches Dekret betr. Gebührensätze für Investitionsregistrierungen vom 15. Mai 1979 (engl. bei Simmonds/Gordon, a.a.O. (Nr. 42) B.2.a., 61-63).

56 Die Handelskammer versichert allerdings in ihrem Kommentar, die Abgaben und Gebühren gem. Art. 26 (d), (e) seien "minimal" (a.a.O. (Nr. 3) 5); derzeit gilt hierfür Gesetz 1213 vom 27. Juni 1967.

57 Malistab sei, die Unternehmen zu schützen, solange sie sich (noch) nicht selbst tragen, wobei jeder Fall einzeln zu würdigen sei (so die Kommentierung, a.a.O., 5). S. allgemein zu solcher Gestaltung Anthoine. a.a.O. (Nr. 50) 5-6; C. H. Fulda/W. F. Schwartz, Regulation of International Trade and Investment (1970) 730-732; ähnlich wie in Kuba Art. 10-12 des jamaikanischen "Industrial Incentives Law (No. 45 of) 1956" (I.L.W. 8: 2 C) oder Art. 15 des südkoreanischen Gesetzes Nr. 1802 vom 3. Aug. 1966 (I.L.W. 12: 2 A).

$58 \mathrm{Im}$ Vergleich dauert die Exemtionsperiode meist ca. 5 Jahre; sie unterscheidet wohl nie zwei oder übersteigt 10 Jahre. Vgl. bsp. Art. 38 der bulgarischen Regelung 1980, Art. 14 des algerischen Invesitionskodex vom 15. Sept. 1966 (engl. in I. L.M. 6 (1967) 92-99), Art. 10 des sudanesischen "Development and Encouragement of Industrial Investment Act, 1974" (I.L.W. $46: 2$ A).

59 Eine durchaus nicht selbstverständliche Regelung; s. etwa Art. 17 des ägyptischen Gesetzes 1974/1977. 
gelung von (ertragsteuerbezogenen) Doppelbesteuerungsabkommen mit den Heimatländern potentieller Unternehmenspartner ${ }^{60}$ dürfte sich zumindest im Normalfall lediglich eine Verschiebung der Steuerbelastung in die überdies durchweg wohl höher besteuernden (Wohn-)Sitz-Staaten der fremden Investoren vollziehen, die allenfalls erst bei der tatsächlichen Ausschüttung von Gewinnen konkrete Gestalt annimmt. Da nun aber nach der Lebenserfahrung jede Auslandsinvestition über kurz oder lang liquidiert wird und zu jenem Zeitpunkt spätestens auch etwa angehäufte Erträge aus dem Gaststaat transferiert werden, dann freilich der Zugriff des Heimatfiskus kaum mehr abzuwehren ist, ${ }^{61}$ mag sich alsbald der bisherige Vorteil als Bumerang erweisen; unilaterale fiktive Steueranrechnung ${ }^{62}$ ist jedenfalls in industrialisierten (Wohn-)Sitz-Staaten nirgends vorgesehen. Zwar mögen manche an Investitionen in Kuba interessierte Unternehmen ihr Vorhaben hinreichend unangreifbar über die Einschaltung verbundener Basisgesellschaften in Niedrigsteuerländern verwirklichen können; ${ }^{63}$ gegenwärtig dürfte aber demungeachtet die Offerte eines (partiellen) Steuerverzichts schwerlich ausschlaggebend für eine Standortwahl im Zuckerinselterritorium sein.

6. Zwiespältige Eindrücke vermittelt die Regelung von Rechtsverfolgung und Rechtsschutz im kubanischen Investitionsregime. Daß in den Gesellschaftsvertrag zwischen kubanischem und fremdem Unternehmen eine Klausel über die Art und Weise sowie auch das Verfahren der Beilegung von Streitigkeiten im Verhältnis untereinander eingehen muß, wurde bereits dargelegt. Obgleich bzw. gerade weil in Art. 13 der Gesetzesverordnung keine weiteren Bestimmungen getroffen sind, bietet sich in Anbetracht der "Internationalität" der konfliktträchtigen Beziehungen die Verabredung internationaler (Handels-)Schiedsgerichtsbarkeit an, erscheint eine derartige Klausel zumindest nicht völlig ausgeschlossen oder unzulässig. ${ }^{64}$ Daneben begründet Art. 35 (2) in bezug auf Wirtschaftsverträge mit dritten kubanischen Organismen die Entscheidungszuständigkeit der staatlichen Schiedsgerichtsbarkeit. ${ }^{65}$ Es fehlt jedoch jede Nor-

60 Auf deren Ergänzungsfunktion weist etwa Kuiper hin (Eur. Tax. 1980, 298).

61 Fast alle Gemeinwesen legen ihrer Steuergesetzgebung das Welteinkommensprinzip zugrunde; dazu bereits $O$. Bühler, Steuerrecht, Internationales, in: K. Strupp/H.-J. Schlochauer, Wörterbuch des Völkerrechts 3 (1962) 377, 380-381; s. ferner H. von Treskow, Förderung ausländischer Direktinvestitionen in Entwicklungsländern durch Steuervergünstigungen (1977) 108-111.

$62 \mathrm{Zu}$ deren Handhabung in bilateralen Verträgen s. $H$. R. Denkl, Die fiktive Steueranrechnung nach den deutschen Doppelbesteuerungsabkommen mit Entwicklungsländern im Einkommen- und Körperschaftssteuerrecht, RIW/AWD 1979, 321-325.

63 Dazu etwa B. Grossfeld, Praxis des Internationalen Privat- und Wirtschaftsrechts (1975) 169-173; V. Kluge, Basisgesellschaften und Doppelbesteuerungsabkommen, RIW/AWD 1975, 525-532.

64 Zur Notwendigkeit aus der Perspektive des Investors s. P. Schlosser. Das Internationale an der internationalen Schiedsgerichtsbarkeit, RIW/AWD 1982, 857, 864-865; K.-H. Böckstiegel, Besondere Aspekte der Wirtschaftsschiedsgerichtsbarkeit mit Parteien aus Entwicklungsländern, NJW 1981, 1862-1867.

Allerdings betonen gerade lateinamerikanische Gemeinwesen seit vielen Jahren die Vorrangigkeit, wenn nicht Ausschließlichkeit der Konfliktlösung durch die nationalen Gerichte des Gaststaates; zu dieser "Calvo"Doktrin vgl. K. Krakau, Lateinamerikanische Doktrinen zur Realisierung staatlicher Unabhängigkeit und Integrität, VRU 8 (1975) 117, 119-129; Foeth, a.a.O. (Nr. 26) 35-40.

65 Deren Rechtsgrundlagen sind in der Handelskammer-Kommentierung aufgeführt (a.a.O. (N. 3) 15); seit 1976 gilt auch in Kuba das "Einheitliche Reglement für Schiedsgerichte bei den Handelskammern der Mitgliedsländer des RGW“ (hierzu D. Pfaff. Vereinheitlichung der Außenhandels-Arbitrage der RGW-Länder und 
mierung der Position gegenüber hoheitlichen Eingriffen, Maßnahmen insbesondere der unmittelbaren zentralen Staatsverwaltung also; hierfür dürfte mithin also gemeines (öffentliches) Recht gelten. ${ }^{66}$ Dabei machen Art. 23, 25 und 26 der kubanischen Verfassung wohl zur Genüge deutlich, daß von konstitutioneller Warte aus betrachtet hier kaum willkürliche oder doch unkalkulierbare Akte zu befürchten sind, ${ }^{67}$ sondern zwar nicht der Bestand, immerhin aber der Wert anerkannten Eigentums verfassungskräftig gewährleistet ist. ${ }^{68}$ Wenngleich nun grundsätzlich von einer Unterwerfung unter die je geltende und anwendbare kubanische Gesetzgebung in bezug auf Verpflichtungen wie auf Rechte auszugehen sein wird, also auch die dort normierten Sanktionen rechtswirksam werden könnten, sollte doch andererseits bei ordnungs-/rechtmäßigem Verhalten aus der konstitutionellen Garantie ein Schutz vor jederzeitigem Widerruf der Vereinigungszulassung gefolgert werden können; ${ }^{69}$ wäre dem nicht so, bliebe ja die Bestimmung in Art. 4 des Gesetzesdekrets weithin Makulatur, wäre die dortige Verknüpfung von Unternehmensdauer und Rentabilität ohne Sinn. Allerdings entspräche es nur der andernorts wohlbekannten Norm für ausländische Investoren, ${ }^{70}$ wenn deren Fehlverhalten auch in vermögensrechtlicher Hinsicht geahndet werden sollte; ein derartiges Vorgehen der einheimischen Behörden ist freilich gerade bei sozialistischen Staaten nach ihrer systemischen Konsolidierung höchst unüblich, ${ }^{71}$ von einem offenkundigen Sonderfall einmal abgesehen. ${ }^{72}$ Bei dergleichen Maßnahmen könnte überdies von (völkerrechtswi-

ihre Auswirkungen auf die internationale Handelsschiedsgerichtsbarkeit im Ost-West-Handel, RIW/AWD 1976, 253, 255-258).

66 Lediglich Art. 24 des Gesetzesdekrets erwähnt in einem Nebensatz den Fall eines einseitigen staatlichen Eingriffs in die Unternehmensaktivitäten. Während in den Investitionsregimes nicht-sozialistischer Entwicklungsländer zumeist Voraussetzungen und/oder Modalitäten hoheitlicher Akte gegenüber den zugelassenen ausländischen Unternehmen näher umschrieben sind (s. nur Art. 7 des ägyptischen Gesetzes von 1974; Art. 4 (d), (e) des philippinischen Gesetzes 1967), hüllt sich die "Joint Venture"-Gesetzgebung fast durchweg in Schweigen (vgl. oben Art. 13 des chinesischen Gesetzes 1979, und dazu Torem/Wohl, Dr. prat. comm. int. 1980, 57; Art. 12 der jugoslawischen Regelung von 1978, und dazu Le Tourneau, Dr. prat. comm. int. 1980, 410-411).

67 Klein (Colum. J. Transnat. L. 1978, 486-487) macht zudem auf die allein dem Strafrecht vorbehaltene Maßnahme der Konfiskation aufmerksam (Art. 59 der Verfassung 1976).

$68 \mathrm{Ob}$ hiernach freilich eine "prompte, adäquate und effektive" Entschädigungspflicht gilt, erscheint überaus zweifelhaft, zumal das vom Völkergewohnheitsrecht kaum (mehr) gefordert wird (vgl. etwa Patzina, a.a.O. (Nr. 38) 38; M. Sornarajah, Compensation for Expropriation: The Emergence of New Standards, J.W.T.L. 13 (1979) 108, 112-113; M. H. Muller, Compensation for Nationalization: A North-South Dialogue, Colum. J. Transnat. L. 19 (1981) 35, 55-78; A. A. Akinsanya, The Expropriation of Multinational Property in the Third World (1980) 25-48; ferner etwa Art. 16 (b) (1) des sudanesischen Gesetzes 1974).

69 S. a. Patzina, a.a.O. (Nr. 38) 18; M. Sornarajah, The Myth of International Contract Law, J.W.T.L. 15 (1981) 187, 216; wie hier wohl auch W. Wengler, JZ 1982, 654-655.

70 Vgl. bsp. Art. 14 des Investitionsgesetzes von Vanuatu (Nr. 2 vom 16. Jan. 1975, I.L.W. X, Vanuatu, 3-15); Art. 26 des "Enterprises Incentives Act " (Nr. 27) von West-Samoa vom 21. Okt. 1965, I.L.W. 55: 2 A); Art. 12, 13 der "Ordonnance-Loi No. 79-027 du 28 septembre 1979 portant Code des Investissements" von Zaire (I.L.W. X, Zaire, 3-15).

71 Florescu, Dr. prat. comm. int. 1978, 249; Scriven, J.W.T.L. 1980, 437.

72 Wie ihn die Aufsage einer Vielzahl erst wenige Jahre zuvor eingegangener industrieller Kooperationsverträge durch chinesische Stellen um die Jahreswende 1980/81 wohl darstellte (s. hierzu etwa HB vom 11. 6. ("Die Anlagenimporteure verschleudern das Geld") und vom 26. 8. 1981 (VR China entschädigt Mitsubishi“), sowie NZZ vom 18.12. 1981 ["Chinesisch-japanische Gespräche in Tokio")). 
driger) Konfiskation o. ä. zumindest dann nicht gesprochen werden, wenn sich durch den Hoheitsakt lediglich ein dem Betroffenen vorhersehbares Risiko verwirklichte. ${ }^{73}$

7. a) Im Gegensatz zu den punktuellen Bestimmungen restriktiver Art bilden die Investitionsanreize der Gesetzesverordnung durchaus ein augenfälliges Ensemble von Regelungen. Obzwar ein Grunderwerb durch Ausländer selbst im Tourismussektor ausgeschlossen bleibt, ${ }^{74}$ dürfte die Standortwahl auf Kuba keine allzu schwierigen (Rechts-)Probleme aufwerfen, sind doch die Möglichkeiten der Anpachtung oder aber des unternehmerischen Zusammenschlusses mit den Land- bzw. Gebäudeeignern eröffnet (Art. 2, 3), im Touristikbereich mag gar ein fremdes Unternehmen direkt als Pächter agieren (2. Sondervorschrift). Die jeweils erforderlichen Kommissions- bzw. Ministerratsgenehmigungen dürfen dabei inhaltlich von der allgemeinen (privatrechtlichen) Regelung abweichen, was kaum zuungunsten des Benutzers erfolgen wird. ${ }^{75}$ Daneben sieht Art. 34 vor, daß die kubanischen Staatsunternehmungen den Wirtschaftsvereinigungen grundlegende infrastrukturelle Lieferungen, überdies all' die (Dienst-)Leistungen verfügbar machen werden, die nicht im Wege des Außenhandels zu erlangen sind. Zwar soll den inländischen staatlichen Unternehmen ein erstes Anrecht zustehen, die wirtschaftlichen Assoziationen mit Energie, Rohstoffen, Materialien und allem übrigen Notwendigen zu versorgen (Art. 32 (a)); diese Option gilt aber nur, soweit ihr Angebot insoweit nach Preis und anderen Konditionen international wettbewerbsfähig ist, ${ }^{76}$ und beläßt den gemeinsamen Unternehmen stets die Freiheit, unmittelbar zu importieren, wessen sie bedürfen (Art. 31 a. E.). Vergleichbar findet sich die Vermarktung der Erzeugnisse im In- und Ausland geregelt: Jede Wirtschaftsvereinigung darf ihre Produkte ohne Einschaltung lokaler Außenhandelsorganisationen ausführen (Art. 31), sie kann sie aber auch inländischen Staatsunternehmen verkaufen, die zur Abnahme gehalten sind, sofern hierdurch ansonsten vorgesehene, den Devisenbestand schmälernde Importe vermieden werden können und die Transaktion "zu international kompetitiven Preisen und anderen Bedingungen" erfolgen soll (Art. 33).

b) Eine nicht gering $\mathrm{zu}$ wertende Vorzugsbehandlung verlautbaren schließlich die arbeitsrechtlichen Regeln des Investitionsdekrets. ${ }^{77}$ Kuba empfiehlt sich hier mit Nachdruck als Niedriglohnland, zumindest was das Gros der Beschäftigten unterhalb der Managementebene anbetrifft - so beträgt das maximale Gehalt eines akademisch

73 Ebenso D.-M. Polter, Auslandsenteignung und Investitionsschutz (1975) 40; Patzina, a.a.O. (Nr. 38) 80; Carreau/Flory/Juillard, a.a.O. (Nr. 16) 545-546.

$74 \mathrm{Zu}$ derartigen weltweit vorfindlichen Normierungen s. insbesondere J. Weisman, Restrictions on the Acquisition of Land by Aliens, A.J.C.L. 28 (1980) 39-66; ferner bsp. Art. 14 (1) der algerischen und Art. 27 der mexikanischen Verfassung sowie Art. 86, 88 EGBGB.

75 S. etwa die of fiziöse Kommentierung (a.a.O. (Nr. 3) 8), wonach es sich um "noch vorteilhaftere" Regelungen handele.

76 Insofern restriktiver Art. 9 (2) der volkschinesischen Gesetzgebung von 1979; s. ferner bsp. Art. 10 (3) (d) des sudanesischen Gesetzes 1974; Art. 18 (1) (a) des ghanesischen "Capital Investment Decree" vom 9. Jan. 1973 (I.L.W. 4 : 2 A); Art. 14 der bolivianischen Gesetzesverordnung von 1965.

77 Hierzu auch die recht ausführliche Kommentierung der Handelskammer (a.a.O. (Nr. 3) 6-8). 
diplomierten Technikers derzeit knapp $400 \$ .{ }^{78}$ Von der inländischen Lohngesetzgebung ausgenommen sind nur die leitenden Angestellten kubanischer Nationalität, deren Bezahlung im Einklang mit der ihrer ausländischen Direktorenkollegen einvernehmlich festgelegt wird (Art. 41). Die Verordnungsbestimmungen enthalten im übrigen auf keiner Ebene der Personalqualifikation zwingende Vorschriften über die - und sei es nur vorrangige - Anstellung einheimischer Arbeitskräfte, ${ }^{79}$ sondern überlassen auch hier die nähere Regelung den Parteien der wirtschaftlichen Vereinigungen (Art. 36 a. E., 44 S. 1); immerhin setzt Art. 36 aber voraus, daß die überwiegende Mehrheit der einfachen Beschäftigten kubanischer Herkunft ist. Nebenkosten entstehen den gemeinsamen Unternehmen - nur diesen - durch das Erfordernis, einen Fonds wirtschaftlicher Anreize für die kubanischen Arbeiter einzurichten und hierfür Beiträge zu leisten (Art. 42); allerdings unterliegen diese Mitteltransfers zum einen nicht der Lohnsummensteuer und sind überdies auch bei der Bemessung der Gewinnsteuer abzugsfähig (Art. 26 (a) 2, (b) 1 a. E.). Durchaus originell erweist sich die Ausgestaltung der Beschäftigungsverhältnisse der Einheimischen, bleiben sie doch auch während der Dauer ihrer Tätigkeit für die Wirtschaftsvereinigung im Dienste ihres bisherigen, kubanischen Arbeitgebers (Art. 38 (1)), werden von diesem Unternehmen zudem weiter entlohnt (Abs. 2), auch und gerade wenn sie aus irgendeinem Grunde nicht mehr für die gemeinsame Unternehmung arbeiten. ${ }^{80}$ In vertragliche Beziehungen treten lediglich die kubanische Einrichtung einerseits und das gemeinsame Unternehmen zum andern, welches für den Einsatz der inländischen Arbeitskräfte einen monatlichen Geldbetrag an die andere Partei entrichtet, der sich in der Höhe nach der Gesamtlohnsumme und anderen geschuldeten Gehaltsleistungen bestimmt (Art. 37). Diese Rahmenkonstruktion führt zunächst dazu, daß über die Anwendbarkeit nationalen Arbeitsrechts für die Leih-Arbeiter kein Zweifel aufkommen, diesen aber im jeweiligen Arbeitsvertrag die Anerkennung der Weisungsbefugnis der anderen Unternehmensleitung deutlich gemacht werden kann. ${ }^{81}$ Weiterhin wird jedoch dieses teils ja vom ausländischen Investor nominierte Direktionspersonal hierdurch in die Lage versetzt, den Arbeitgeber und Vertragspartner zum Austausch unfähiger oder -williger Beschäftigter zu veranlassen, ist doch die Überlassung einer disziplinierten und produktiven "Mannschaft" wesentlicher Bestandteil der Absprache, nicht zuletzt angesichts des generell für ein Entwicklungsland überdurchschnittlichen Bildungs- und Ausbildungsstandes der kubanischen Arbeitsbevölkerung. ${ }^{82}$

78 Vgl. die ebd., 7, wiedergegebene Lohnskala.

79 Auch andernorts zeigt sich hierin eine gewisse Lockerung; vgl. etwa die argentinischen Gesetze 19.151 (vom 5. Aug. 1971, engl. in I.L.M. 10 [1971] 1194-1200) und 20.557 (1973), nach deren Art. 14 bzw. 5 (g) $85 \%$ der Beschäftigten die Nationalität des Gastlandes besitzen mußten, mit der Regelung in Art. 41 (1) (h) der Ausführungsverordnung 283/77.

80 So ausdrücklich der offiziöse Kommentar (a.a.O. (Nr. 3) 6), der an anderer Stelle (ebd., 4) die "absolute Freiheit" der gemeinsamen Unternehmen auch bei der Bestimmung der Zahl wie bei der Auswahl der Beschäftigten hervorhebt.

81 In aller Regel fungiert in sozialistischen Staaten hingegen das "Joint Venture" selbst als Arbeitgeber (s. Art. 30, 35 der bulgarischen Regelung von 1980; Art. 32, 34 des rumänischen Dekrets Nr. 424/1972).

$82 \mathrm{Vgl}$. etwa den Weltentwicklungsbericht 1979 der I.B.R.D., Tabelle 23 (188-189, 205). 
c) Eine Unterwerfung unter nationales Recht kommt in Art. 39 zum Ausdruck, der zur Einhaltung der geltenden Vorschriften betr. Arbeitssicherheit und Hygiene verpflichtet; aus derartigen Einzelbestimmungen (s. a. Art. 3 (2), 6, 7 S. 1) mag auf eine allgemeine Inländergleichbehandlung ${ }^{83}$ zu schließen sein, wenn nicht sogar (vgl. Art. 25, 30, 31, 43) Privilegien für ausländische Unternehmen rechtsverbindlich niedergelegt sind.

8. Ist die Existenz der Wirtschaftsvereinigung angesichts des Ablaufs der vereinbarten Wirksamkeitsdauer oder aber durch hoheitliche Eingriffe gefährdet oder liegen sonst Gründe für die Beendigung der (inländischen) Aktivitäten vor - nach Maßgabe des Art. 8 (3) der Gesetzesverordnung sollten die Assoziationsstatuten Näheres regeln -, so bleibt die Abwicklung von Auflösung und Liquidation ausschließlich Sache der Beteiligten, ist lediglich eine Anzeige an das Handelsregister vonnöten (Art. 12). In den seltensten Fällen will freilich ein ausländischer Teilhaber sein Kapital auch weiter im Lande belassen, wie ihm gleichermaßen an einer möglichst reibungslosen Repatriierung von Gewinnen gelegen ist. ${ }^{84}$ Das kubanische Regime - in Gestalt des Banco Nacional als zuständigem Organ - garantiert hier Transferfreiheit ohne ersichtliche Beschränkungen, abgesehen davon, daß ein Teil der Gewinne vorerst zur Rücklagenbildung verwendet werden muß (Art. 22). Insonderheit sehen Art. 23, 24 des Gesetzesdekrets die Rücküberweisung in konvertiblen Währungen vor, kennen zudem keinerlei Vorbehalt für Situationen nationaler Zahlungsbilanzschwierigkeiten, wie er selbst in manch' bilateralem Kapitalschutzabkommen Erwähnung fand. ${ }^{85}$

9. In den Schlußbestimmungen findet sich letztlich aber eine ggf. recht weit reichende Beschränkung des Anwendungsbereichs des Investitionsregimes; es gilt nicht für grenzüberschreitende Kompensationsgeschäfte, ${ }^{86}$ worunter auch die neuen, "technischen" Formen internationaler Investitionen ${ }^{87}$ wie etwa die Errichtung schlüsselfertiger Anlagen fallen $^{88}$ (4. Sondervorschrift, (a)). Diese Bestimmung enthält zwar nichts inhaltlich

83 Zu diesem "Standard" des herkömmlichen Fremdenrechts s. etwa I. Brownlie, Principles of Public International Law ( $\left.{ }^{3} 1979\right)$ 521-528; E. Menzel/K. Ipsen(/E. Wehser), Völkerrecht ( $\left.{ }^{2} 1979\right)$ 175-177.

$84 \mathrm{Zu}$ völkergewohnheitsrechtlichen Grenzen für die Ausübung staatlicher Währungshoheit in bezug hierauf s. Z. A. Kronfol, Protection of Foreign Investment (1972) 51; H. Gattiker, Behandlung und Rolle von Auslandsinvestitionen im modernen Völkerrecht: Eine Standortbestimmung, Schw. Jb. Int. R. 37 (1981) 25 , 31; F. A. Mann, The Legal Aspect of Money ( $\left.{ }^{4} 1982\right)$ 465-466.

85 So spricht etwa der schwedisch-chinesische Investitionsschutzvertrag vom 29. März 1982 (I.L.M. 21 (1982) 477-478) in Art. 5 von einer Repatriierung "ohne ungebührliche Verzögerung", und das Protokoll zum deutschen Abkommen mit Papua Neuguinea (vom 12. Nov. 1980, BGBI. 1982 II, 390) setzt in Nr. 7 "unverzüglich" mit "binnen zwei Monaten" gleich. Vgl. ferner die Kritik Manns (a.a.O. [Nr. 84] $529 \mathrm{Nr}$. 83) an der häufig verwendeten britischen Ausnahmeklausel, wie sie bsp. in Art. 7 (3) der Abmachung mit den Philippinen (vom 3. Dez. 1980, Cmnd. 8148, I.L.M. 20 (1981) 326-328) zu finden ist.

86 Dazu jüngst J. I. Walsh, Countertrade: Not just for East-West any more, J.W.T.L. 17 (1983) 3-11; eingehend M. Fontaine, Aspects juridiques des contrats de compensation, Dr. prat. comm. int. 7 (1981) 179-223; sowie das Sonderheft über ein von der F.E.D.U.C.I. veranstaltetes Kolloquium "Les contrats de compensation industrielle", Dr. prat. comm. int. 8 (1982) 157-383.

$87 \mathrm{Vgl}$. bereits $P . K a h n$, Etat actuel du droit des investissements étrangers dans les pays en voie de développement, in: M. Bos (Hrsg.), The Present State of International Law and Other Essays (1973) 283, 301-304.

88 S. etwa $M$. Tuts, L'évolution des contrats "clé en main", Dr. prat. comm. int. 3 (1977) 633-636; $M$ Salem/M.-A. Sanson, Les contrats "clé en main" et les contrats "produit en main" (1979). 
Außergewöhnliches, grenzt aber wohl erstmals im nationalen Rahmen den Regelungsgegenstand ausdrücklich auf klassische Direktinvestitionstypen ein ${ }^{89}$ Klarstellende Bedeutung trägt auch die nachfolgende Vorschrift, die wahrhaft internationale, auf Völkerrecht gründende ${ }^{90}$ Vereinigungen mit kubanischer und fremder Beteiligung ebenfalls nicht dem Investitionsregime unterwirft.

\section{Schluß}

Ein Versuch, das kubanische Gesetzesdekret bereits kurz nach Inkrafttreten zu würdigen, muß notwendig Fragment bleiben; jedoch wird bereits jetzt dessen Regelungen ein beträchtliches Maß an Sachverstand und Ausgewogenheit attestiert werden dürfen. Mag eine solche Wertung auch die in einem sozialistischen Gemeinwesen selten fehlenden bürokratischen Implementierungshemmnisse ${ }^{91}(\mathrm{zu})$ leicht nehmen, so kann doch gegenüber solchen Bedenken auf die eindeutige Fassung von für den ausländischen Investor essentiellen Bedingungen und Gewährleistungen hingewiesen werden. Die Entscheidung für die Vertragsform als Modalität der Investitionsgestaltung bietet zudem dem fremden Unternehmenspartner die reelle Möglichkeit, die eigene (Vermögens-)Position verbindlich ab- und einzugrenzen, ja wohl gar eine gewisse "Stabilisierung" der Rechtslage in bezug auf das je bestimmte Projekt zu erreichen.

Aus der Sicht des Gaststaats hingegen läßt sich in der Tat von einer behutsamen Offnung gegenüber der (privaten) internationalen Wirtschaft sprechen, ist doch die im Inland tätig werdende ökonomische Assoziation in vielfacher Weise staatlicher, wenn auch meist nur indirekter Einflußnahme und Kontrolle zugänglich ${ }^{92}$ und behält sich der Staat letztendlich stets die Zulassung einer ausländischen Kapitalbeteiligung vor ${ }^{93}$ Zudem unterliegt die Republik Kuba insbesondere westlichen (Industrie-)Staaten gegen-

89 Vgl. auch Carreau/Flory/Juillard, a.a.O. (Nr. 16) 457-458; Hahn/Gramlich, Arch VR 1983, 196-203; sowie schon L. Kopelmanas, La protection des investissements privés à l'étranger, Dr. prat. comm. int. 1 (1975) 3, 8-10.

90 Hierzu bereits B. Goldman, Le droit des sociétés internationales, J.D.I. (Clunet) 90 (1963) 320-389; P. Kahn. International Companies, J.W.T.L. (1969) 498-521; Grossfeld, a.a.O. (Nr. 63) 216-226.

$91 \mathrm{Vgl}$. bsp. C. E. Coughlin, An Economic Analysis of Yugoslav Joint Ventures, J.W.T.L. 17 (1983) 12, 17-31; NZZ vom 11. 10. 1980 ("Dornenvolle jugoslawische Kooperationsgesetzgebung"); Süddeutsche Zeitung vom 18. 1. 1982 ("Japaner werfen in Rumänien das Handtuch"); FT vom 10. 10. 1980 ("China's first foreign joint venture suspended"); HB vom 16.8. 1982 ("Peking feilt an der Kooperationsbasis").

92 Vgl. für eine durchaus ähnliche Rechtslage J. Marciniuk, Selbständige Wirtschaftstätigkeit ausländischer Investoren in Polen, RIW/AWD 1980, 467, 469-470.

93 Es kann also keineswegs von einer "Freiheit zum Investieren" für Ausländer die Rede sein, wie sie etwa im Raum der Europäischen (Wirtschafts-) Gemeinschaft rechtliche Gestalt angenommen hat (vgl. Art. 58, $67 \mathrm{ff}$., 221 EWGV; s. zu territorialer Erweiterung auch $H$. Jouan jean, Les mouvements de capitaux dans le cadre de l'adhésion de la Grèce à la Communauté européenne, R.M.C. 243 (1981) 14-20). 
über nur sehr dünnen völker(vertrags)rechtlichen Bindungen, ${ }^{94}$ ist in bezug auf die Ausübung ihrer ständigen Souveränität über die eigenen (Natur-)Schätze ${ }^{95}$ sohin überaus frei und durch Sätze des internationalen Gewohnheitsrechts schwerlich an je für wünschenswert erachteten Maßnahmen gegen ausländische Unternehmensteilhaber gehindert. ${ }^{96}$ Bislang jedenfalls blieb mithin der Kniefall vor dem Kapital(ismus) aus!

94 Immerhin gehört der Karibik-Staat sowohl dem GATT als auch der W.I.P.O. und überdies mehreren internationalen Schiedsgerichtsübereinkommen an (s. die Auflistung in der Handelskammer-Publikation, a.a.O. (Nr. 3) 16-17).

95 Aus dem schier unübersehbaren Schrifttum s. aus jüngerer Zeit etwa G. Elian, The Principle of Sovereignty over Natural Resources (1979); H. Reinhard, Rechtsgleichheit und Selbstbestimmungsrecht der Völker in wirtschaftlicher Hinsicht (1980); F. Rigaux, Pour un autre ordre international, in: J. Blanc/F. Rigaux, Droit économique 2 (1979) 269, 343-357.

96 Selbst wenn die Auffassung von Sir Gerald Fitzmaurice zuträfe, eine Stabilisierungsklausel in Verträgen zwischen Staaten und ausländischen Investoren sei ein wirksames Mittel gegen Expropriationsakte (sep. op. im Schiedsverfahren zwischen der kuwaitischen Regierung und der American Independent Oil Company; s. den Spruch vom 24. März 1982, I.L.M. 21 (1982) 976-1042/53, insbesondere 1050-1053 - ohne nähere Begründung ebenso das Erkenntnis eines I.C.S.I.D.-Tribunals vom 30. Nov. 1979 in Sachen Agip Company ./. Volksrepublik Kongo, I.L.M. 21 (1982) 726, 735), und eine solche Abrede auch der "Joint Venture"-Gesetzgebung nicht gänzlich fremd sein mag (s. Art. 6 des jugoslawischen Gesetzes von 1978; dazu Coughlin. J.W.T.L. 1983, 16-17), so bedarf es doch jedenfalls einer entsprechenden verbindlichen Zusage durch den Gaststaat selbst, iure imperii. Erlal.3 und Handhabung eines autonom gesetzten Investitionsregimes allein können noch kaum völkerrechtliche Verpflichtungen bzw. Haftungstatbestände begründen (s. aber $J$. Stoll. Vereinbarungen zwischen Staat und ausländischem Investor (1981) 120-127; andererseits J. Kuusi, The Host State and the Transnational Corporation (1979) 154, 165, und nunmehr. P. Kahn. Contrats d'Etat et nationalisation, J.D.I. (Clunet) 109 (1982) 844, 848-861). 
ment policies. Active popular participation is described as an indispensable element in basic-needs oriented rural development. Participation in turn requires political and administrative decentralization. Some comments on restrictions on decentralization in developing countries are given in conclusion of the theoretical considerations.

The last section discusses in some detail the strategic value of deconcentrating the administration to improve implementation capacity. Empirical evidence is drawn from the Zambian Decentralization Programme of January 1st, 1981.

\title{
Guerilla Movements in Latin America
}

\author{
By Peter Waldmann
}

The article discusses the conditions of growth and success of guerilla movements in Latin America. It is centred around three topical complexes. First, the principal causes of revolutionary upheaval in Nicaragua under the leadership of the Sandinists are described. In order to test the wider validity of the causal factors delineated with regard to Nicaragua, two further movements are analysed: Venezuela in the early 1960s, and Argentina in the first half of the 1970s. As a result of this comparison, five main conditions are identified for the success, of failure, of guerilla movements in Latin America. The final chapter examines the significance of the Sandinist victory in Nicaragua for the political and military situation in Central America; some conclusions are then offered on the prospects of the conflicts in El Salvador and Guatemala.

\section{Cuba's Legislative Decree No. 50/1982 - Castro's Genuflection Before Capitalism?}

\section{By Ludwig Gramlich}

Cuba enacted a legislative decree on foreign investment in early 1982. Its 50 sections may have been influenced by other joint venture legislation in socialist countries all over the world, but also by genuine Latin American conceptions. The Cuban decree contains rather detailed and relatively liberal provisions for foreign investors which, however, have to be completed by acts of public international law. Particularly in the field of taxation, unilateral fiscal incentives may sooner or later be maintained by tax treaties with home countries of foreign enterprises.

Another significant trait of the new Cuban foreign investment regulations are some provisions on labour law distinguishing between permanent contractual relations of Cuban employers and employees on the one side and temporary work of those employees under direction of foreign entrepreneurs. 
The scope of the decree docs not cover types of foreign investment other than direct investment; co-operation agreements and similar contractual arrangements will be treated under a special regime. It must not be overlooked that the Cuban regulations do not in any way mean that foreign investors will enjoy a guaranteed or stabilized position under public international law. Therefore every foreign investor has to remain aware of possible future changes in Cuba's investment climate. 